\title{
Trends in Pneumonia and Influenza-associated Hospitalizations in South Korea, 2002-2005
}

\author{
Soon Ae Kim', Paul E. Kilgore', Sang-Yi Lee ${ }^{2}$, Batmunkh Nyambat', and Moran Ki³ \\ 'Division of Translational Research, International Vaccine Institute, Seoul, South Korea (Present affiliation: Department of Pharmacy \\ Practice, Eugene Applebaum College of Pharmacy and Health Sciences, Wayne State University, Detroit, Michigan, USA), ${ }^{2}$ Korean \\ National Health Insurance Corporation, Seoul, South Korea (Present affiliation: Department of Health Policy and Management, \\ School of Medicine, Cheju National University, Jeju, South Korea), and ${ }^{3}$ Department of Preventive Medicine, \\ Eulji University College of Medicine, Daejeon, South Korea
}

\begin{abstract}
Pneumonia and influenza are leading causes of morbidity and mortality across the globe. Korea has established the national health-insurance system to cover the entire Korean population since 1989. The aim of this study was to describe the epidemiologic trends in pneumonia and influenza-associated hospitalizations and deaths using the Korean National Health Insurance databases and national vital statistics. During 2002-2005, 989,472 hospitalizations and 10,543 deaths due to pneumonia and influenza were recorded. Eighty-one percent of the hospitalizations were related to diagnoses with unspecified aetiology. The average annual rate of hospitalizations due to pneumonia and influenza was 5.2 per 1,000 people [95\% confidence interval (CI) 5.2-5.3], and the hospitalization rate increased by $28 \%$ (from 4.5 to 5.8 per 1,000 people) during the four-year study period. In addition, deaths due to pneumonia and influenza increased by $48 \%$ (2,829 during 2003, 3,522 during 2004, and 4,192 during 2005). Overall, the national burden of hospitalizations and deaths due to pneumonia and influenza in Korea was high, and it increased for all age-groups during the study period. A comprehensive review of potential interventions by the government authorities should aim to reduce the burden of pneumonia and influenza.
\end{abstract}

Key words: Hospitalization; ICD-10 codes; Influenza; National health insurance; Pneumonia; South Korea

\section{INTRODUCTION}

Globally, pneumonia and influenza are leading causes of morbidity and mortality $(1,2)$. In 2005, the United Nations Children's Fund (UNICEF) and the World Health Organization (WHO) reported that an estimated 1.6 million people die of pneumococcal disease every year, including 700,000 to one million children aged less than five years (2). More than $70 \%$ of patients with pneumonia were found in Africa and Asia (3). Each year, an estimated $5-10 \%$ of adults and $20-30 \%$ of children become ill due to influenza. In addition, 250,000-500,000 deaths and 3-5 million severe influenza cases, which occur each year, are associated with seasonal epidemics of influenza (4). In the USA, there are more than 1.6 million annual hospitali-

Correspondence and reprint requests should be addressed to:

Dr. Soon Ae Kim

International Vaccine Institute

SNU Research Park, San 4-8 Nakseongdae-Dong

Kwanak Gu, Seoul 151-919

South Korea

Email: sakim@ivi.int

Fax: +82-2-872-2803 zations attributable to pneumonia and influenza, of which $65 \%$ have pneumonia or influenza as the primary diagnosis (5-7). Each year in the USA, over 36,000 deaths are attributable to pneumonia and influenza.

With growing recognition of the impact of pneumonia and influenza, prevention through vaccination has been recommended for both children and adults but the vaccine uptake has been low in resource-limited countries $(8,9)$. While available vaccines have been shown to reduce the burden of pneumonia and influenza, the choice of antimicrobial therapy is likely to diminish case fatality, duration of hospitalization, and the frequency of clinical sequelae (10-13). In South Korea, the Health Institute for Review and Assessment Service (HIRA) and the National Health Insurance Corporation (NHIC) reported that organism-unspecified pneumonia was the fifth leading cause of inpatient health-insurance claims during 2005 (14). However, as of 2010, limited epidemiologic information regarding the recent trends in pneumonia and influenza in Korea is available.

In this study, we describe the burden of hospitalizations due to pneumonia and influenza and their epi- 
demiologic trends using national health-insurance and national mortality databases. Results of this analysis will be useful to support the development of improved public-health policies for the control of pneumonia and influenza in Korea.

\section{MATERIALS AND METHODS}

The National Health Insurance Programme reimburses providers for medical services, and the national health-insurance system covers $97 \%$ of the Korean population through mandatory contributions from insured residents. An additional 3\% of the Korean population is covered through the Korean Medical Aid Programme which provides healthcare to low-income households $(14,15)$.

We accessed the national health-insurance databases from January 2002 through December 2005, which contain the patient's date of birth, sex, International Classification of Diseases, $10^{\text {th }}$ Revision (ICD10) codes for primary and secondary discharge diagnoses, dates of admission and discharge, type of hospital (primary, secondary, or tertiary), and the patient's address. Medical institutions receive medical service-fees from the Government by issuing reimbursement claims every month. For any patient with multiple hospital admissions, records were combined and considered the same disease episode if the date of admission was less than 30 days following the most recent date of discharge. To ensure confidentiality of patient information during analysis, individual national registration numbers were replaced with an unrelated unique eight-digit identification number assigned by the data-management staff of the Health Policy Research Institutes, NHIC.

For this analysis, a hospitalization was defined by admission to an inpatient facility for 24 hours or more. Records of hospitalizations due to pneumonia and influenza were included for analysis, if the ICD-10 diagnostic codes for pneumonia and influenza were listed for either the primary or the secondary diagnosis. The ICD-10 codes are a valid method for the diagnosis of pneumonia in a previous study (16). The diagnostic codes were classified based on unspecified (i.e. a clinical syndrome with no mention of pathogen) and specific diagnoses (i.e. the pathogen is mentioned in diagnosis). We grouped the following diagnoses with unspecified aetiology as presumed infectious and non-infectious pneumonia (B05.2, J12.8 to J12.9, J15.8 to J15.9, J16.8, J17.0 to J17.3, J178, J18.0 to J18.1, J18.8 to J18.9, and J18.2) and bronchitis (J20.0, J20.1). Aetiology-specific causes included in this analysis are as follows: Haemophilus influenzae-associated pneumonia (J14), influenza (J10.0 to J10.1, J11.0, J11.1,
J10.8, and J11.8), Mycoplasma pneumoniae (J15.7), Streptococcus pneumoniae-asscoated pneumonia (J13), other bacterial pneumonias (A54.8, J15.0 to J15.6, and J16.0), and other viral pneumonias (B012, and J12.0 to J12.2). We also accessed the national vital statistics from the Korea National Statistical Office (http://www.kosis.kr) to describe the patterns of deaths for the 2003-2005 period. Using the same list of pneumonia and influenza ICD-10 diagnostic codes applied in the analysis of hospitalization records, we identified deaths due to pneumonia and influenza by analyzing the primary and secondary causes of death.

In Korea, the Government conducts a national census every five years. Projected population data are available for years that occur during the intercensus periods. The government census office performs population projection estimates based on births, deaths, and immigrations using cohort component methods (www.nso.go.kr). To calculate the annual and age-specific rates of hospitalizations, national population projections for 2002 through 2004 were used as denominators. We also adjusted for hospitalization rate using the national census data of 2005 during the study period.

Analysis of data was performed using the SAS statistical software (version 9.12) (SAS Institute, Inc., Cary, North Carolina, USA) and the Stata software (version 11.0) (StataCorp LP, Texas, TX, USA).

\section{RESULTS}

\section{General characteristics and trends by age-group and diagnosis}

During 2002-2005, there were 21,781,400 all-cause hospitalizations among all age-groups in Korea. This total included 4,753,009 hospitalizations during 2002, 5,399,067 during 2003, 5,706,573 during 2004, and 5,922,751 during 2005. During the fouryear study period, we identified 989,472 hospitalizations due to pneumonia and influenza among all age-groups in Korea. The proportion of hospitalizations due to pneumonia and influenza ranged from $4.5 \%$ to $4.6 \%$ during $2002-2005$, and the proportion of deaths associated with pneumonia and influenza ranged from $1.1 \%$ to $1.7 \%$ of all deaths during 2003-2005 (Table 1). Of all the hospitalizations, we found that $81.1 \%$ of patients $(n=802,462)$ were admitted to tertiary-care hospitals.

Of all the pneumonia and influenza-associated hospitalizations, 48.4\% ( $\mathrm{n}=478,751)$ occurred among children aged less than five years. An additional $21.2 \%(n=208,143)$ occurred among persons aged $5-49$ years, and $21.5 \%(n=212,615)$ occurred among the elderly aged 65 years and above. Among all the 


\begin{tabular}{|cccccccc|}
\hline \multicolumn{2}{|c|}{ Table 1. Characteristics of hospitalizations and deaths due to pneumonia and influenza in Korea, } \\
$2002-2005$
\end{tabular}

age-groups, males (55.9\%) were more often hospitalized but this difference was most pronounced in the first three decades of life and in persons aged over 60 years $(\mathrm{p}<0.05)$. Most $(81.3 \%$; $n=804,116)$ pneumonia and influenza-associated hospitalizations were coded with diagnoses with unspecified aetiology. Of these, $55.9 \%(n=448,986)$ occurred among children aged less than 15 years. Of all the hospitalizations due to pneumonia and influenza, $18.7 \%(\mathrm{n}=185,356)$ were associated with aetiologyspecific diagnoses. Of these, $64.2 \%(\mathrm{n}=118,922)$ were associated with M. pneumoniae, $20.8 \%$ $(\mathrm{n}=38,563)$ with other pneumonia-causing bacteria, 9.1\% $(\mathrm{n}=16,885)$ with influenza viruses, $3.9 \%(n=7,219)$ with other viruses that cause pneumonia, $1.8 \%(\mathrm{n}=3,358)$ with $S$. pneumoniae, and $0.2 \%(\mathrm{n}=409)$ with $H$. influenzae. Mycoplasma was frequently diagnosed among hospitalized children aged less than five years (17.3\%) and children aged less than 15 years $(27.2 \%)$ while influenza was frequently diagnosed among adults aged $15-49$ years (Table 2 and Fig. 1).

The number of deaths due to pneumonia and influenza increased by $48.2 \%$ from 2,829 in 2003 to 3,522 in 2004 and 4,192 in 2005. Most (98.1\%) deaths due to pneumonia and influenza were associated with presumed infectious pneumonia during 2003-2005 (2,777 in 2003; 3,459 in 2004; and 4,110 in 2005) while the number of deaths caused by influenza was very limited from 20 in 2003 to 38 in 2004 and 6 in 2005.

\section{Rates of hospitalizations and mortality}

During 2002-2005, the monthly rate of hospitalizations due to pneumonia and influenza increased significantly ( $\mathrm{p}=0.038$ ) (Fig. 2). A peak of hospitalizations due to pneumonia and influenza occurred in the final weeks of 2002, and two broader peaks occurred in the spring of 2004 and 2005. In contrast, the mortality rate declined in the first six months of 2003 and then increased steadily from 2003 through 2005.

The monthly distribution of hospitalizations due to pneumonia and influenza also varied by agegroup. In the combined four-year database, the burden of diseases in children had two seasonal peaks (Fig. 3A and 3B)—one during the autumn and winter months (October to January) and the second one during the spring (April to June). Hospitalizations among children aged 5-14 years (Fig. 3B and 3C) showed a winter seasonal peak with longer duration and a shorter spring peak than children aged less than five years. Young adults aged 1549 years and middle-aged adults (50-64 years) (Fig. $3 \mathrm{D}$ and $3 \mathrm{E}$ ) experienced smaller and shorter winter epidemics but the spring epidemics in these agegroups showed higher peaks and longer durations compared to the elderly. Persons aged 65 years and above (Fig. 3F) had a large, sustained year-round burden of hospitalizations due to pneumonia and influenza, with several smaller peaks and durations in all the years $(\mathrm{p}<0.05)$.

For all the hospitalizations due to pneumonia and influenza, the mean duration of stay was 9.6 patient-days (median 7 days). By age-group, the median duration (days) of stay increased with age from six days among children aged less than five years to 9.5 days in the elderly aged 65-74 years and 10 days among those aged $\geq 75$ years (data not shown).

The average annual rate of hospitalizations due to pneumonia and influenza was 5.2 per 1,000 people (95\% CI 5.2-5.3) in all ages during the study period. During 2002-2005, the overall rate of hospitalizations increased by $27.5 \%$ from 4.5 per 1,000 people to 5.8 per 1,000 people (Table 3 ). Children aged less than five years (37.5 per 1,000 people) and the elderly aged $\geq 75$ years (20.3 per 1,000 people) had the highest age-group-specific hospitalization rates for pneumonia and influenza over the four-year study period. When we adjusted the hospitalization rate using the 2005 census data, children aged less than five years and the elderly aged $\geq 65$ years showed the increased annual rates of hospitalizations due to pneumonia and influenza while young children and adults aged 5-49 years had the similar adjusted annual hospitalization rates dur- 


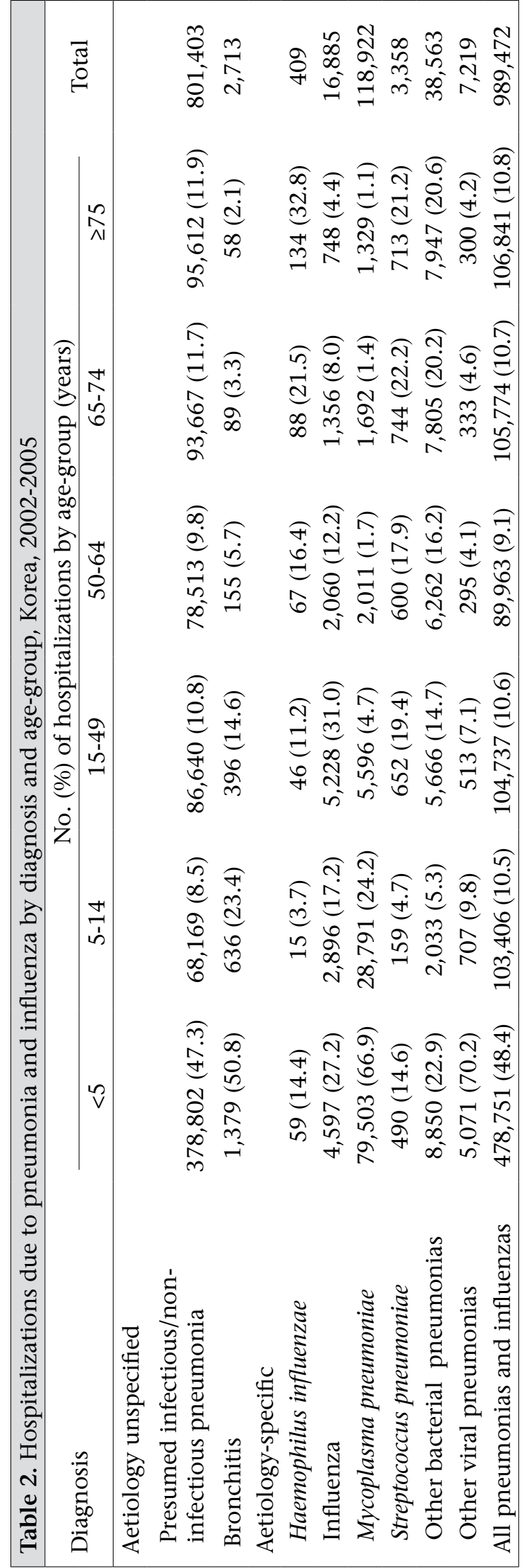

ing the four-year study period. The hospitalization rate among the elderly aged $\geq 75$ years increased by $92.4 \%$ (from 13.4 to 25.7 per 1,000 people) and by $13.6 \%$ among children aged less than five years (from 43.5 to 49.4 per 1,000 people). As the age increased, the percentage of increase in the hospitalization rates over the study period rose dramatically from $30.2 \%$ among those aged $50-64$ years to $51.1 \%$ for those aged $65-74$ years and $92.4 \%$ for patients aged $\geq 75$ years.

\section{DISCUSSION}

In Korea, the increased number and rate of hospitalizations due to pneumonia and influenza and the increased number of deaths in all age-groups were observed despite the introduction of pneumococcal vaccine in 2003 and the introduction of influenza vaccine in $1997(17,18)$. In addition, rates of hospitalizations due to pneumonia and influenza and mortality were high among very young and elderly persons. The burden of hospitalizations due to pneumonia and influenza among the elderly has increased sharply with the ageing of the Korean population. Over the four-year study period, the rate of hospitalizations due to pneumonia and influenza among those aged 75 years and above increased by $92.4 \%$. From a national perspective, the dramatic rise in the rates of hospitalizations due to pneumonia and influenza is of concern as the population in this age-group increased by $19 \%$ during the same period. The NHIC reported that the total cost of hospitalizations for the elderly aged 65 years or above increased faster $(62 \%)$ than the total number of hospitalizations (19\%) nationwide over the four-year period from 2002 to 2005 (19). These demographic trends observed in Korea mirror those found in other developed nations of Asia and Europe (20). Nevertheless, population shifts alone are unlikely to entirely explain the rise in hospitalizations due to pneumonia and influenza. Given the recent trends in diseases, it is likely that a substantial proportion of hospitalizations due to pneumonia and influenza in the elderly occurred among those with the underlying medical conditions. It has been particularly recognized that chronic medical conditions may place the elderly at a higher risk for serious pneumonia and influenza $(15,21)$. In 2005 , Korea spent $6 \%$ of its gross domestic product (GDP) on healthcare, onethird less than the $9 \%$ of GDP health expenditure averaged among 30 economically-developed nations (22). Nevertheless, the increasing burden of hospitalizations due to pneumonia and influenza in Korea underscores the massive costs of these diseases to society and the healthcare system. These observations suggest that public-health strategies 


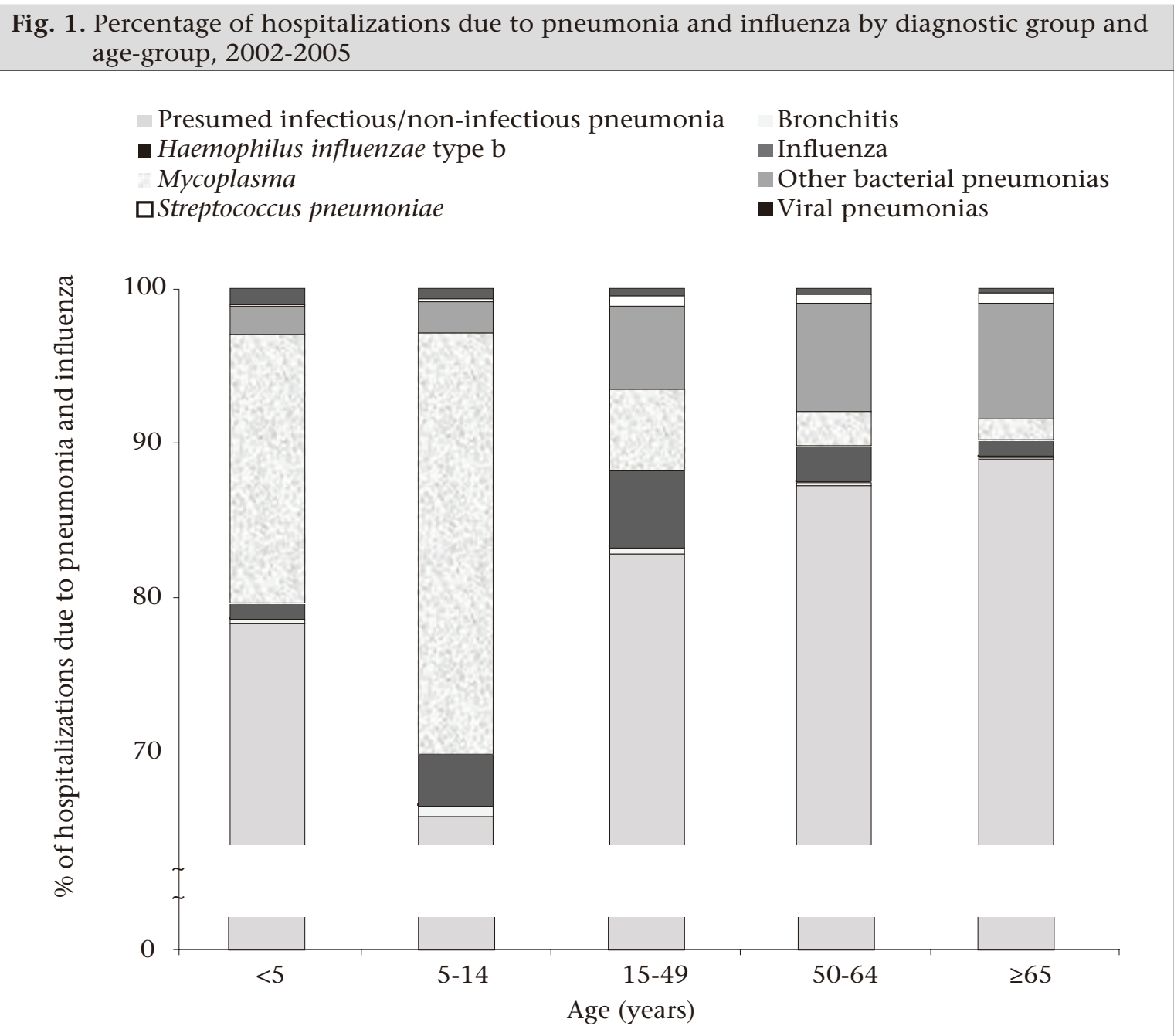

to prevent pneumonia and influenza are likely to be cost-effective in Korea.

A few comparable national studies were conducted that allow for age-group comparisons. Elsewhere, there is a strong evidence that hospitalizations due to pneumonia and influenza occur at high rates. For example, in Spain, the annual hospitalizations due to pneumonia and influenza among the people aged 50-64 years occured in 143 cases per 100,000 people (23). In England, the annual hospitalizations due to pneumonia episodes ranged from 10 to 15 per 1,000 people, with the hospital admission rates of $1-4$ per 1,000 people per year among children aged $\leq 5$ years (24). A study of all-cause community-acquired pneumonia among patients aged above 14 years in Spain reported an overall hospitalization rate of 1.62 per 1,000 people (25). In 2005, Burgner and Richmond reported a pneumonia-related hospitalization rate of 5-8 per 1,000 person-years among Australian children aged less than five years (26). Results of an analy-sis of the
US Medicare insurance records showed a high rate of hospitalizations due to pneumonia and influenza among the elderly (15.1-23.4 per 1,000 people), with a 55\% increase in hospitalization over a 13-year period (27). We also found that children aged less than five years had the highest age-specific rate $(37.5$ per 1,000$)$ of hospitalizations due to pneumonia and influenza among all age-groups. In our study, the hospitalizations rate for childhood pneumonia was higher compared to the mean annual excess influenza-associated hospitalizations among Hong Kong children (1999 data: <1-year old, 28.8 per 1,000; 1-2-year old, 20.9 per 1,000; and 2-5-year old, 7.7 per 1,000 children) (28). We also found that hospitalizations due to pneumonia and influenza were distributed with the distinct winter and spring peaks in all age-groups. These classical winter and spring peaks of pneumonia and influenza are consistent with data from Australia (26), Spain (29), the USA $(30,31)$, Brazil (32), and Viet Nam (33). The year-to-year variations observed in the seasonal pneumonia and influenza- 
Fig. 2. Monthly distribution of hospitalization rates, 2002-2005 and mortality rates, 2003-2005 due to pneumonia and influenza

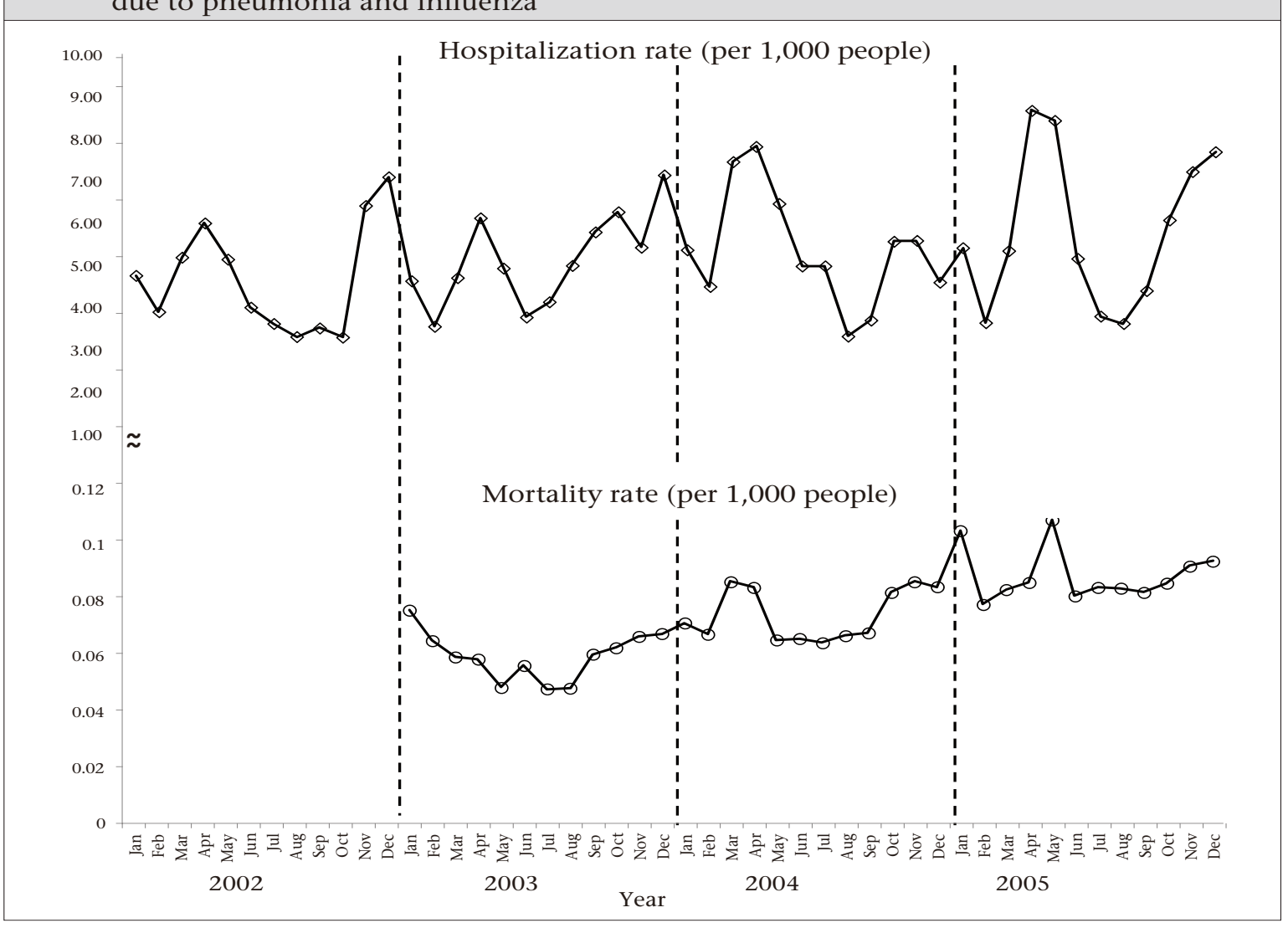

Fig. 3. Weekly distribution of hospitalizations due to pneumonia and influenza by age-group, 2002-2005

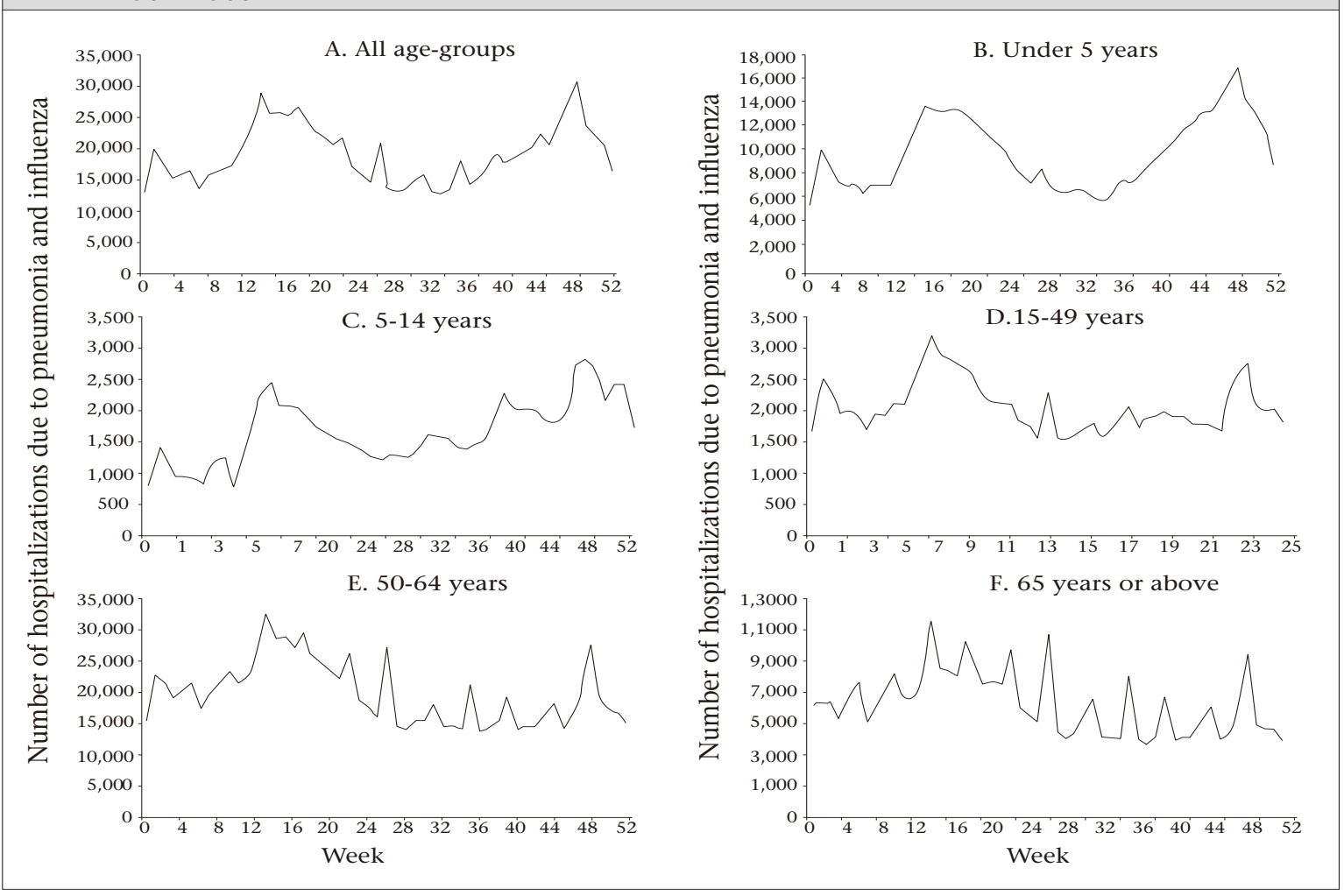




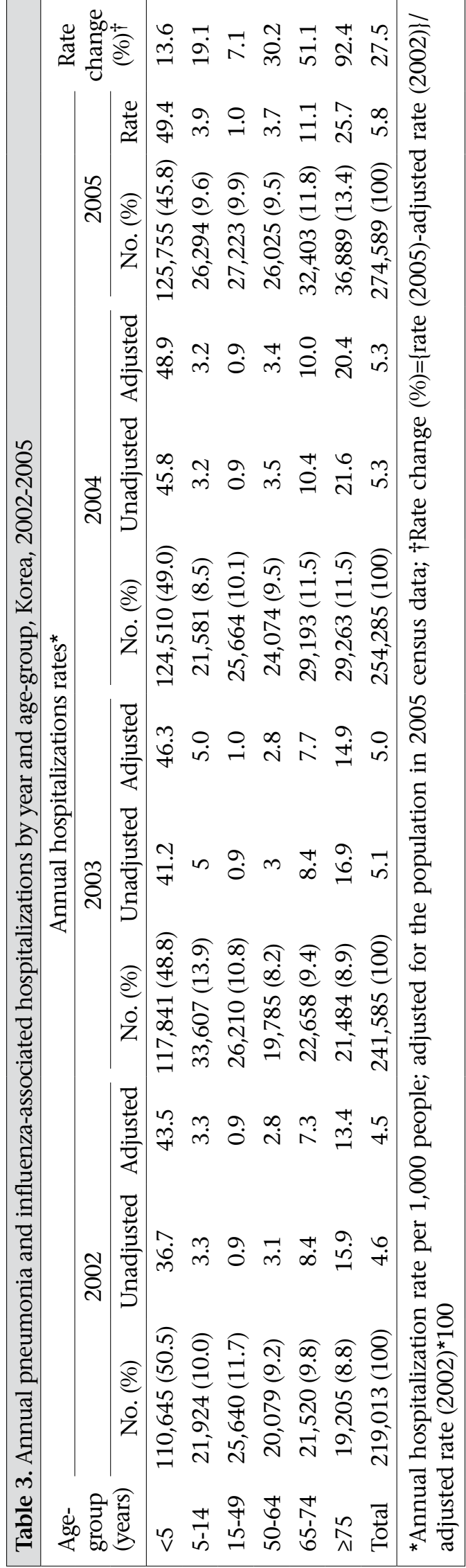

associated hospitalization peaks may be due to annual variations in the circulating influenza virus subtypes or other pathogens found in Korea. The laboratory-based national influenza surveillance system reported predominantly H3N2 influenza virus isolates in the 2002-2003, 2003-2004 and 20042005 influenza seasons, which likely influenced the seasonal peak distribution observed in this analysis. The pneumonia and influenza-associated hospitalization peak that started in the winter of 2005 was larger than previous years and was associated with a new influenza subtype H1N1 (34).

\section{Limitations}

This study has some limitations. As with other studies of administrative hospitalization databases, the potential for diagnostic misclassification must be considered. In this study, we were unable to identify individual patients and then conduct a validation of diagnoses in the hospital patientcharts. Nonetheless, it is unlikely that patients who presented with signs and symptoms of pneumonia and influenza were misclassified into non-pneumonia and influenza diagnostic categories given the distinct clinical nature of lower respiratory tract disease (35-37). We recognize that our analysis may include patients with nosocomially-acquired pneumonia and influenza. To reduce the potential for including nosocomial pneumonia, we removed records where the date of diagnosis occurred after the date of admission prior to our final analysis. In addition, we did not evaluate cohort effects that may influence age- and time-related patterns of pneumonia and influenza using regression analysis (7). Such analysis will be considered in future analysis that will include additional years of data. In future analysis, laboratory-confirmed diagnoses obtained from the national surveillance systems may be linked to hospitalization records in the national health-insurance databases to validate ICD-10 diagnoses. In addition, we recommend further studies of the burden of pneumonia and influenza in Korea that may begin by comparing hospitalizations before and after the introduction of pneumococcal and influenza vaccines into the national immunization programmes.

\section{Conclusions}

The growing toll of hospitalizations due to pneumonia and influenza in Korea is of concern. Such trends underscore the need for a comprehensive review of preventive health services that may reduce the burden of pneumonia and influenza. This review of services in Korea should consider prospective evaluation of the impact and cost-effectiveness of vaccination. Given the epidemiologic profile of hospitalizations due to pneumonia and influ- 
enza in these national data, it is likely that health policies focused on the prevention of pneumonia and influenza will provide important opportunities for reducing the total burden of pneumonia and influenza among the Korean population.

\section{ACKNOWLEDGEMENTS}

The authors thank I.S. Park, Division of Statistical Analysis, National Insurance Corporation Research Institute, for technical support and assistance for data management. Robert Holman, Office of Director, Division of Viral and Rickettsial Diseases, National Center for Infectious Diseases, Centers for Disease Control and Prevention, assisted with data analysis, interpretation, and review of the manuscript. They also thank Kathy Murray, a freelance technical editor, for editorial assistance.

\section{REFERENCES}

1. Centers for Disease Control and Prevention. Pneumonia and influenza death rates, United States, 19791994. MMWR Morb Mortal Wkly Rep 1995;44:535-7.

2. United Nations Children's Fund. Pneumonia: the forgotten killer of children. New York, NY: United Nations Children's Fund, 2006:7.

3. Singh V. The burden of pneumonia in children: an Asian perspective. Paediatr Respir Rev 2005;6:88-93.

4. World Health Organization. Influenza. Fact sheet no. 211. Revised March 2003. Geneva: World Health Organization, 2003. (http://www.who.int/mediacentre/ factsheets/fs211/en, accessed on 11 March 2007).

5. Molinari NA, Ortega-Sanchez IR, Messonnier ML, Thompson WW, Wortley PM, Weintraub E et al. The annual impact of seasonal influenza in the US: measuring disease burden and costs. Vaccine 2007;25:508696.

6. Thompson WW, Shay DK, Weintraub E, Brammer L, Cox N, Anderson LJ et al. Mortality associated with influenza and respiratory syncytial virus in the United States. JAMA 2003;289:179-86.

7. Cohen SA, Klassen AC, Ahmed S, Agree EM, Louis TA, Naumova EN. Trends for influenza and pneumonia hospitalization in the older population: age, period, and cohort effects. Epidemiol Infect 2010;138:113545 .

8. Smith NM, Bresee JS, Shay DK, Uyeki TM, Cox NJ, Strikas RA; Advisory Committee on Immunization Practices. Prevention and control of influenza: recommendations of the Advisory Committee on Immunization Practices (ACIP). MMWR Recomm Rep 2006;55:1-42.

9. Mahoney RT, Krattiger A, Clemens JD, Curtiss R, 3rd. The introduction of new vaccines into developing countries. IV: Global Access Strategies. Vaccine
2007;25:4003-11.

10. Kollef $\mathrm{MH}$. Inadequate antimicrobial treatment: an important determinant of outcome for hospitalized patients. Clin Infect Dis 2000;31(Suppl 4):S131-8.

11. McGeer A, Green KA, Plevneshi A, Shigayeva A, Siddiqi N, Raboud J et al. Antiviral therapy and outcomes of influenza requiring hospitalization in Ontario, Canada. Clin Infect Dis 2007:45:1568-75.

12. Lee N, Chan PK, Choi KW, Lui G, Wong B, Cockram CS et al. Factors associated with early hospital discharge of adult influenza patients. Antivir Ther 2007;12:501-8.

13. Nichol KL, Nordin JD, Nelson DB, Mullooly JP, Hak E. Effectiveness of influenza vaccine in the communitydwelling elderly. N Engl J Med 2007;357:1373-81.

14. Health Insurance Review and Assessment Service. Annual report of national health insurance statistics. Seoul: Health Insurance Review and Assessment Service, 2006.

15. Cho KW, Chung Y, Roh YK Cho B, Kim CH, Lee HS. Health care for older persons: a country profile-Korea. J Am Geriatr Soc 2004;52:1199-204.

16. Skull SA, Andrews RM, Byrnes GB, Campbell DA, Nolan TM, Brown GV et al. ICD-10 codes are a valid tool for identification of pneumonia in hospitalized patients aged $>$ or $=65$ years. Epidemiol Infect 2008;136:232-40.

17. Lee WJ. [Establishment of guidelines for immunization against Streptococcus pneumoniae in Korea]. Seoul: Korea Center for Disease Control, 2006;10:85-92. (Korean).

18. Kee SY, Lee JS, Cheong HJ, Chun BC, Song JY, Choi WS. Influenza vaccine coverage rates and perceptions on vaccination in South Korea. J Infect 2007;55:27381.

19. Park JYKI, Kim KH. [Service for the elderly of longterm care management system in Korea]. Seoul: National Health Insurance Corporation, Republic of Korea, 2006:76. (National Health Insurance Corporation report no. 2006-11). (Korean).

20. Thompson WW, Shay DK, Weintraub E, Brammer $\mathrm{L}$, Bridges $\mathrm{CB}$, Cox NJ et al. Influenza-associated hospitalizations in the United States. JAMA 2004; 292:1333-40.

21. Hebert PL, McBean AM, Kane RL. Explaining trends in hospitalizations for pneumonia and influenza in the elderly. Med Care Res Rev 2005;62:560-82.

22. Organisation for Economic Co-operation and Development. OECD health at glance 2007. Paris: Organisation for Economic Co-operation and Development, 2007:90. (http://www.oecd-ilibrary.org/docserver/ download/fulltext/8107051e.pdf?expires $=13198648$ $84 \&$ id=id\&accname $=$ guest $\&$ checksum $=813 \mathrm{CEC} 4 \mathrm{DB}$ 
30A8203700352D25A09B2CF, accessed on 28 October 2011).

23. Gil A, Gil R, Oyaguez I, Carrasco P, Gonz Lez A. Hospitalization by pneumonia and influenza in the 5064 year old population in Spain (1999-2002). Hum Vaccin 2006;2:181-4.

24. Farha T, Thomson AH. The burden of pneumonia in children in the developed world. Paediatr Respir Rev 2005;6:76-82.

25. Almirall J, Bolíbar I, Vidal J, Sauca G Coll P, Niklasson $\mathrm{B}$ et al. Epidemiology of community-acquired pneumonia in adults: a population-based study. Eur Respir J 2000;15:757-63.

26. Burgner D, Richmond P. The burden of pneumonia in children: an Australian perspective. Paediatr Respir Rev 2005;6:94-100.

27. Fry AM, Shay DK, Holman RC, Curns AT, Anderson LJ. Trends in hospitalizations for pneumonia among persons aged 65 years or older in the United States, 1988-2002. JAMA 2005;294:2712-9.

28. Chiu SS, Lau YL, Chan KH, Wong WH, Peiris JS. Influenza-related hospitalizations among children in Hong Kong. N Engl J Med 2002;347:2097-103.

29. Lenglet AD, Hernando V, Rodrigo P, Larrauri A, Donado JD, de Mateo S. Impact of flu on hospital admissions during 4 flu seasons in Spain, 2000-2004. BMC Public Health 2007;7:197.

30. Thompson WW, Comanor L, Shay DK. Epidemiology of seasonal influenza: use of surveillance data and statistical models to estimate the burden of disease. $J$
Infect Dis 2006;194(Suppl 2):S82-91.

31. Greene SK, Ionides EL, Wilson ML. Patterns of influenza mortality among US elderly by geographic region and virus subtype, 1968-1998. Am J Epidemiol 2006;163:316-26.

32. Alonso WJ, Viboud C, Simonsen L, Hirano EW, Daufenbach LZ, Miller MA. Seasonality of influenza in Brazil: a traveling wave from the Amazon to the subtropics. Am J Epidemiol 2007;165:1434-42.

33. Nguyen H, Saito R, Ngiem H, Nishikawa M, Shobugawa Y, Nguyen D et al. Epidemiology of influenza in Hanoi, Vietnam, from 2001 to 2003. J Infect 2007;55:58-63.

34. Korea Centers for Disease Control and Prevention. Korean Influenza Surveillance Scheme. Influenza summary update. Seoul: Korea Centers for Disease Control and Prevention, 2007. (http://dis.cdc.go.kr/ Influenza/Information/week_view.asp, accessed on 17 March 2007).

35. Ives DG, Samuel P, Psaty BM, Kuller LH. Agreement between nosologist and cardiovascular health study review of deaths: implications of coding differences. $J$ Am Geriatr Soc 2009;57:133-9.

36. Stein RT, Marostica PJ. Community-acquired pneumonia: a review and recent advances. Pediatr Pulmonol 2007;42:1095-103.

37. Gedeborg R, Furebring M, Michaëlsson K. Diagnosis-dependent misclassification of infections using administrative data variably affected incidence and mortality estimates in ICU patients. J Clin Epidemiol 2007;60:155-62. 